\title{
FLUX DEPENDENCE OF PERSISTENT CURRENT \\ IN A MESOSCOPIC DISORDERED TIGHT BINDING RING
}

\author{
J.Heinrichs \\ Institut de Physique B5, University of Liege, \\ Sart-Tilman, B-4000 Liege, Belgium
}

\begin{abstract}
We reconsider the study of persistent currents in a disordered one-dimensional ring threaded by a magnetic flux $\phi$, using he one-band tight-binding model for a ring of $N$-sites with random site energies. The secular equation for the eigenenergies expressed in terms of transfer matrices in the site representation is solved exactly to second order in a perturbation theory for weak disorder and fluxes differing from half-integer multiples of the flux quantum $\phi_{0}=h c / e$. From the equilibrium currents associated with the one-electron eigenstates we derive closed analytic expressions for the disorder averaged persistent current for even and odd numbers, $\mathrm{Ne}$, of electrons in the ground state. Explicit discussion for the half-filled band case confirms that the persistent current is periodic with a period $\phi_{0}$, as in the absence of disorder, and that its amplitude is generally suppressed by the effect of the disorder. In comparison to previous results, based on an approximate analysis of the secular equation, the current suppression by disorder is strongly enhanced by a new periodic factor proportional to $1 / \sin ^{2} \frac{2 \pi \phi}{\phi_{0}}$, for $\phi \neq$ (integer) $\phi_{0} / 2$.
\end{abstract}

PACS numbers: 71.50.+t, 72.15.Rn, 72.15.-v, 72.20.-i 


\section{INTRODUCTION}

An Aharonov-Bohm flux threading a metallic or semiconducting ring leads to a persistent equilibrium current even if the ring is disordered, provided it is of mesoscopic size smaller than the mean free path for inelastic scattering. This is a direct consequence of quantum coherence which is not destroyed by elastic impurity scattering. The persisitent current is periodic in the magnetic flux $\phi$ with a period given by the elementary flux quantum $\phi_{0}=h c / e$ (with $h$ the Planck constant, $c$ the speed of light and $-e$ the electron charge). The existence of persistent currents was predicted for one-dimensional rings by BŸttiker, Imry and Landauer[1] and has later been observed experimentally in real three-dimensional rings by several groups[2-4]. Detailed theoretical investigations of the persistent current have appeared for onedimensional systems as well as for multichannel two- and three-dimensional rings [5-28]. Several reviews of the subject have also been published[29-31].

Many of these studies were motivated by the experiment of Levy et al.[2] whose result for the persistent current averaged over an ensemble of $10^{7}$ disordered copper rings is several orders of magnitude larger than the averaged current calculated for models taking only the scattering by random impurities into account $[14,16]$. This led to attempts of including, in addition, the effect of the Coulomb interaction between the diffusing electrons[15,13,20,23,26], which improved the agreement between measured and calculated persistent currents considerably. However, in other recent numerical studies it was found that Coulomb interactions suppress the persistent current in one-dimensional disordered systems, rather than enhancing it $[21,24,25]$.

The present paper concerns the study of persistent currents in a one-dimensional disordered tight binding ring threaded by a magnetic flux, in the absence of electronelectron interaction. We recall that in previous discussions, starting with the key paper of BŸttiker et al.[1], the effect of the flux is described via the flux-modified boundary condition (with $x$ the position along the one-dimensional ring of circumference $L$ )

$$
\psi(x+L)=e^{i 2 \pi \frac{\phi}{\phi_{0}}} \psi(x)
$$

for the ground state wavefunction of the system in the absence of flux[32,33]. This description of the flux has been demonstrated for the Schršdinger equation in continuous space where the vector potential may be eliminated by choosing a gauge in which the external magnetic field vanishes in the interior of the ring [32,33].

We consider a tight-binding system composed of $N$ one-orbital atomic sites

of spacing $a$ on a ring threaded by a magnetic flux in the direction perpendicular to the ring. This system is described by the set of difference equations

$$
\begin{aligned}
& -e^{i \frac{2 \pi}{N} \frac{\phi}{\phi_{0}}} \varphi_{n+1}-e^{-i \frac{2 \pi}{N} \frac{\phi}{\phi_{0}}} \varphi_{n-1}+\varepsilon_{n} \varphi_{n}=E \varphi_{n}, n=2,3, \ldots, N-1 \\
& -e^{i \frac{2 \pi}{N} \frac{\phi}{\phi_{0}}} \varphi_{2}-e^{-i \frac{2 \pi}{N} \frac{\phi}{\phi_{0}}} \varphi_{N}+\varepsilon_{1} \varphi_{1}=E \varphi_{1} \\
& -e^{i \frac{2 \pi}{N} \frac{\phi}{\phi_{0}}} \varphi_{1}-e^{-i \frac{2 \pi}{N} \frac{\phi}{\phi_{0}}} \varphi_{N-1}+\varepsilon_{N} \varphi_{N}=E \varphi_{N}
\end{aligned}
$$


which correspond to the familiar Anderson model for a disordered ring modified by the flux of the external vector potential[6]. Here $\varphi_{n}$ is the amplitude of an eigenstate wavefunction at site $n, E$ and $\varepsilon_{n}$ are the corresponding eigenvalue and random site energies (potentials) in units of minus a constant nearest-neighbour hopping parameter.

For clarity's sake we now briefly rederive the boundary conditions (1) for the discrete ring described by equations (2-4). To this end we perform a gauge transformation

$$
\psi_{n}=e^{i \frac{2 \pi}{N} \frac{\phi}{\phi_{0}} n} \varphi_{n}, n=1,2, \ldots, N
$$

to new amplitudes $\psi_{n}$, which transforms the system (2-4) into

$$
\begin{aligned}
& -\psi_{n+1}-\psi_{n-1}=\left(E-\varepsilon_{n}\right) \psi_{n}, n=2,3, \ldots, N-1, \\
& -\psi_{2}-e^{-i 2 \pi \frac{\phi}{\phi_{0}}} \psi_{N}=\left(E-\varepsilon_{1}\right) \psi_{1}, \\
& -e^{i 2 \pi \frac{\phi}{\phi_{0}}} \psi_{1}-\psi_{N-1}=\left(E-\varepsilon_{N}\right) \psi_{N},
\end{aligned}
$$

where the effect of the flux is transferred entirely to the bond linking the sites 1 and $N$. These sites are bounding the continuous sequence of sites $n=2,3, \ldots, N-1$ described by the equations (6) from which the flux is eliminated. We now observe that the system (6-8) is formally equivalent to

$$
-\psi_{n+1}-\psi_{n-1}=\left(E-\varepsilon_{n}\right) \psi_{n}, n=1,2, \ldots, N
$$

with the conditions (where (10.b) is the analog of (1) taken at the origin)

$$
\begin{gathered}
\psi_{N+1}=e^{i 2 \pi \frac{\phi}{\phi_{0}}} \psi_{1}, \\
\psi_{N}=e^{i 2 \pi \frac{\phi}{\phi_{0}}} \psi_{0},
\end{gathered}
$$

which describe a flux-free system defined by the $N$ equations (9), involving $N+2$ amplitudes $\psi_{n}$, in which the flux enters via the boundary conditions (10.a,b). Indeed, these boundary conditions ensure that the equations (9) for $n=1, N$ coincide with (7-8). In addition, one sees that rotating all amplitudes in (9) once around the ring $\left(\psi_{m} \rightarrow \psi_{m+N}, m=1,2, \ldots, N\right)$ leaves these equations unchanged under the boundary condition

$$
\psi_{m+N}=e^{i 2 \pi \frac{\phi}{\phi_{0}}} \psi_{m}
$$

This shows that the flux modified (or twisted) boundary condition (1) is also valid for the discrete ring. We further recall that the similarity between the boundary 
condition (11) and the property of Bloch functions in a periodic potential[1] (with $\frac{2 \pi}{L} \frac{\phi}{\phi_{0}}$ playing the role of the momentum and the ring size $L$ corresponding to the size of the unit cell) implies a flux periodicity with period $\phi_{0}$ of the eigenstates and eigenenergies, and hence of the equilibrium persistent current carried by an energy level $E_{n}$,

$$
I_{n}=-c \frac{\partial E_{n}}{\partial \phi}
$$

Cheung et al. [5] have studied the persistent current in the system described by equation (9) and (10.c) in terms of a two steps process: in the first step the flux-free ring is being cut open and deformed int o a linear chain whose Schršdinger equation (9) is transformed to a tight-binding Bloch wave basis [5,35] and is solved in terms of a transfer matrix for amplitudes of Bloch waves travelling to the right and to the left of the chain; in the second step the wave amplitudes at the two ends of the chain are further matched by imposing the flux modified boundary condition (10.c). This leads to an exact secular equation for the eigenenergies of the ring involving only the flux and the total amplitude transmission coefficient, $t$, of the disordered chain of the first step at energy $E$. Cheung et al. combine their secular equation with (11) to calculate the persistent current, by making simple ad hoc assumptions about the energy dependence of $t$ and approximating the effect of the disorder in the averaged transmission coefficient in terms of the Landauer formula [5]. In the case of weak disorder they find a constant, flux-independent, reduction of the amplitude of the persistent current with respect to its value for a perfect ring.

From a first-principles point of view the constant reduction of the current amplitude due to the disorder obtained by Cheung et al. [5] is surprising. After all, the exact explicit expressions of the transmission- and reflection coefficients, $t$ and $r$, are complicated functions of the random site energies $\varepsilon_{j}$ and of energy (through the dependence on the Bloch wavenumber, $k=\arccos (-E / 2))[5,35]$, from which one may suspect the existence of a non trivial interplay of disorder and flux in persistent currents in the exact eigenstates.

The purpose of this paper is precisely to discuss an exact first principles analytic calculation of the eigenenergies and persistent currents for the ring described by (2-4) in the framework of a second order perturbation theory for weak disorder. In particular, we wish to study quantitatively the effect of the expected interplay of disorder and magnetic flux on the magnitude of the persistent current. On the other hand, a way of assessing the relative importance of electron interaction effects in persistent currents $[15,13,20,21,23-26]$ in the disordered regime, is by comparing numerical calculations with results obtained for non-interacting electrons. Exact analytical results for the effect of disorder on the persistent current for non interacting electrons, such as those discussed in this paper, are clearly valuable in this context.

The perturbation theory for the eigenenergies of the ring for weak disorder is conveniently developed by starting from a secular equation expressed in terms of transfer matrices for the Schršdinger equation (2-4) [or (6-8)] in the site representation. The secular equation for the eigenenergies of the ring as well as their explicit determination to second order in the random site energies are discussed in Sect. II. In Sect. III we use the results of Sect. II for calculating the persistent current averaged over the disorder for a system of $N_{e}$ spinless particles in the ground state of the tight-binding system. Our results will thus be relevant to average currents 
obtained from measurements for a large number of weakly disordered mesoscopic rings corresponding to different realizations of the disorder[2]. The discussion of our results for the typical low filling $\left(N_{e} \ll N\right)$ and half-filled band $\left(N_{e}=\frac{N}{2}\right)$ cases, their comparison with previous work and some concluding remarks are presented in Sect. IV. 


\section{TRANSFER MATRIX PERTURBATION THEORY}

Our aim is to calculate the electronic eigenenergies of the tight-binding ring described by (2-14) to successive orders in small random fluctuations $\varepsilon_{i}$ of the site energies about a zero mean value. This amounts to choosing the energy level of the individual atomic sites as the zero of energy. We find that the method based on tranfer matrices in the site-space representation[34,35] is ideally suited for this purpose since these matrices depend linearly on the energies $\varepsilon_{i}$.

Defining the unimodular transfer matrices

$$
\hat{P}_{n}=\left(\begin{array}{cc}
-\left(E-\varepsilon_{n}\right) & -e^{-i \alpha} \\
e^{i \alpha} & 0
\end{array}\right), \alpha=\frac{2 \pi}{N} \frac{\phi}{\phi_{0}}, n=1,2, \ldots, N \quad,
$$

we have, from (2-4),

$$
\begin{aligned}
& \left(\begin{array}{c}
\varphi_{n+1} \\
\varphi_{n}
\end{array}\right)=e^{-i \alpha} \hat{P}_{n}\left(\begin{array}{c}
\varphi_{n} \\
\varphi_{n-1}
\end{array}\right), n=2,3, \ldots, N-1 \\
& \left(\begin{array}{c}
\varphi_{2} \\
\varphi_{1}
\end{array}\right)=e^{-i \alpha} \hat{P}_{1}\left(\begin{array}{c}
\varphi_{1} \\
\varphi_{N}
\end{array}\right) \\
& \left(\begin{array}{c}
\varphi_{1} \\
\varphi_{N}
\end{array}\right)=e^{-i \alpha} \hat{P}_{N}\left(\begin{array}{c}
\varphi_{N} \\
\varphi_{N-1}
\end{array}\right) .
\end{aligned}
$$

By combining the amplitude vector of components $\varphi_{N}$ and $\varphi_{N-1}$, obtained by iterating (13) in terms of the vector with components $\varphi_{2}$ and $\varphi_{1}$, with (14 15), we readily obtain the following exact equation for the eigenvalues of the ring:

$$
\operatorname{detm}\left(e^{-i 2 \pi \frac{\phi}{\phi_{0}}} \prod_{n=1}^{N} \hat{P}_{n}-\hat{1}\right)=0,
$$

which simplifies to

$$
\operatorname{tr}\left(\prod_{n=1}^{N} \hat{P}_{n}\right)=2 \cos 2 \pi \frac{\phi}{\phi_{0}}
$$

since the determinant of the product of unimodular matrices, $\prod_{n=1}^{N} \hat{P}_{n}$, is equal to unity.

For the purpose of our perturbation treatment for the eigenvalues for weak disorder we first discuss the solutions of (17) in the absence of disorder. In this case

$$
\prod_{n=1}^{N} \hat{P}_{n} \equiv \hat{P}^{N}, \text { with } \hat{P}=\left(\begin{array}{cc}
-E^{0} & -e^{-i \alpha} \\
e^{i \alpha} & 0
\end{array}\right)
$$


where $E^{0}$ denotes eigenenergies of the perfect ring. In order to determine the elements of the matrix $\hat{P}^{N}$ we diagonalize $\hat{P}$ by means of a similarity transformation. The eigenvalues of $\hat{P}$ are

$$
\lambda_{1,2}=\frac{1}{2}\left(-E^{0} \pm \sqrt{E^{02}-4}\right)
$$

which may be rewritten in the form

$$
\lambda_{1,2}=e^{ \pm i s}
$$

with

$$
E^{0}=-2 \cos s
$$

The similarity transformation which diagonalizes $\hat{P}$ is defined by the non-unitary eigenvector matrix

$$
\hat{U}=\left(\begin{array}{cc}
e^{i(s-\alpha)} & e^{-i(s+\alpha)} \\
1 & 1
\end{array}\right), \text { with } \hat{U}^{-1}=\frac{e^{i \alpha}}{2 i \sin s}\left(\begin{array}{cc}
1 & -e^{-i(s+\alpha)} \\
-1 & e^{i(s-\alpha)}
\end{array}\right)
$$

Using the fact that $\left(\hat{U}^{-1} \hat{P} \hat{U}\right)_{i j}=\lambda_{i} \delta_{i, j}, i=1,2$, we then obtain from (21) and (22),

$$
\hat{P}^{m}=\hat{U}\left(\hat{U}^{-1} \hat{P} \hat{U}\right)^{m} \hat{U}^{-1}=\frac{1}{\sin s}\left(\begin{array}{cc}
\sin (m+1) s & -e^{-i \alpha} \sin m s \\
e^{i \alpha} \sin m s & -\sin (m-1) s
\end{array}\right)
$$

In the absence of disorder the eigenvalue equation (17) then reduces to

$$
\cos N s=\cos \frac{2 \pi \phi}{\phi_{0}}
$$

which leads to the energy levels

$$
E_{k}^{0}(\phi)=-2 \cos \frac{2 \pi}{N}\left(k+\frac{\phi}{\phi_{0}}\right),
$$

using (21), and to the corresponding equilibrium currents (equation (11))

$$
I_{k}^{0}=-\frac{2 e}{N \hbar} \sin \frac{2 \pi}{N}\left(k+\frac{\phi}{\phi_{0}}\right),
$$

where $k=0, \pm 1, \pm 2, \ldots$ These results coincide with those obtained earlier by Cheung et al.[5] for the perfect tight-binding ring. For levels with $|k| \ll N$ the energies (25) as a function of $\phi / \phi_{0}$ grow quadratically with $\phi / \phi_{0}$ away from minima at $\phi / \phi_{0}=-k, k=0, \pm 1, \ldots$ For such levels therefore, the energy spectrum is similar to the familiar free particle spectrum composed of intersecting parabolas 
whose ordinates increase as a function of $\phi / \phi_{0}$ away from their respective vertices at $0, \pm 1, \pm 2, \ldots[5]$.

We proceed with the solution of (17) for weak random fluctuations of the site energies where we expand the equation to successive orders in the $\varepsilon_{i}$ and obtain the corresponding first and second orders corrections, $E_{k}^{(1)}$ and $E_{k}^{(2)}$, for the perturbed energy levels,

$$
E_{k}=E_{k}^{0}+E_{k}^{(1)}+E_{k}^{(2)}+\cdots, k=0, \pm 1, \ldots .
$$

The transfer matrices $\hat{P}_{n}$ are split into unperturbed and perturbed parts:

$$
\hat{P}_{n}=\hat{P}+\hat{V}_{n} \equiv \hat{P}-\left(\Delta E-\varepsilon_{n}\right)\left(\begin{array}{ll}
1 & 0 \\
0 & 0
\end{array}\right),
$$

where

$$
\Delta E=E^{(1)}+E^{(2)}+\cdots,
$$

and the matrix product, $\prod_{n=1}^{N} \hat{P}_{n}$, is expanded to quadratic order in the quantities $\Delta E-\varepsilon_{n}$. This yields

$$
\begin{aligned}
\prod_{n=1}^{N} \hat{P}_{n}=\hat{P}^{N}+\sum_{m=1}^{N} \hat{P}^{m-1} \hat{V}_{m} & \hat{P}^{N-m} \\
& +\sum_{n=2}^{N} \sum_{m=1}^{n-1} \hat{P}^{m-1} \hat{V}_{m} \hat{P}^{n-m-1} \hat{V}_{n} \hat{P}^{N-n}+\cdots
\end{aligned}
$$

By inserting this expression in (17), using (23), and equating the two sides of the equation order by order we obtain, after some straightforward algebra,

$$
E_{k}^{(1)}=E^{(1)}=\frac{1}{N} \sum_{n=1}^{N} \varepsilon_{n}
$$

and

$$
\begin{aligned}
E_{k}^{(2)}=\frac{1}{N \sin q_{k} \sin N q_{k}} \sum_{n=2}^{N} \sum_{m=1}^{n-1}\left(E^{(1)}-\varepsilon_{m}\right)\left(E^{(1)}-\varepsilon_{n}\right) . & \sin (n-m) q_{k} \cdot \sin (N-n+m) q_{k}
\end{aligned}
$$

where

$$
q_{k}=\frac{2 \pi}{N}\left(k+\frac{\phi}{\phi_{0}}\right)
$$

Clearly, the weak disorder perturbation theory breaks down at flux values equal to half-interger multiples of the flux quantum $\phi_{0}$ where (32) diverges. Such divergencies are familiar[19] in other perturbative studies of persistent currents[13,14,16]. 


\section{AVERAGED PERSISTENT CURRENT}

The random eigenenergy corrections (31) and (32) are easiest to analyse through low order moments. Here we shall consider only the averaged eigenenergies and the corresponding averaged persistent currents. These are relevant to experiments obtaining the persistent currents in a large number of identical mesoscopic rings corresponding to different realizations of the disorder[2]. In averaging the eigenenergies (27) we assume a site energy disorder in the form of gaussian white noise with a correlation

$$
\left\langle\varepsilon_{i} \varepsilon_{j}\right\rangle=\varepsilon_{0}^{2} \delta_{i, j}
$$

From (31) and (32) we thus obtain $\left\langle E_{k}^{(1)}\right\rangle=0$ and

$$
\left\langle E_{k}^{(2)}\right\rangle=-\frac{\varepsilon_{0}^{2}}{N^{2} \sin q_{k} \sin N q_{k}} \sum_{n=2}^{N} \sum_{m=1}^{n-1} \sin (n-m) q_{k} \sin (N-n+m) q_{k}
$$

By performing the finite summations over sites in (35) we finally get

$$
\left\langle E_{k}^{(2)}\right\rangle=\frac{\varepsilon_{0}^{2}}{4 \sin q_{k}}\left[\left(1+\frac{1}{N}\right) \cot N q_{k}-\frac{1}{N} \cot q_{k}\right], k=0, \pm 1, \pm 2, \ldots
$$

From (11), (25) and (36) we then obtain the averaged current from the level $E_{k}$

$$
\begin{aligned}
\left\langle I_{k}\right\rangle & =-\frac{e}{N \hbar}\left\{2 \sin q_{k}-\frac{N \varepsilon_{0}^{2}}{4 \sin q_{k}} .\right. \\
& {\left.\left[\left(1+\frac{1}{N}\right)\left(\frac{1}{\sin ^{2} \frac{2 \pi \phi}{\phi_{0}}}+\frac{1}{N} \cot \frac{2 \pi \phi}{\phi_{0}} \cot q_{k}\right)+\frac{1}{N^{2}}\left(1+2 \cot ^{2} q_{k}\right)\right]\right\}, }
\end{aligned}
$$

which reduces to

$$
\begin{aligned}
\left\langle I_{k}\right\rangle=-\frac{e}{N \hbar}\left[2 \sin \frac{2 \pi}{N}\left(k+\frac{\phi}{\phi_{0}}\right)-\frac{N \varepsilon_{0}^{2}}{4 \sin ^{2} \frac{2 \pi \phi}{\phi_{0}}} \frac{1}{\sin \frac{2 \pi}{N}\left(k+\frac{\phi}{\phi_{0}}\right)}\right] \\
k=0, \pm 1, \ldots,
\end{aligned}
$$

by dropping terms of order $\frac{1}{N}$ and $\frac{1}{N^{2}}$ in the square brackett of (37).

In order to obtain the total averaged persistent current $\langle I\rangle$ for a system of $N_{e}$ electrons in the ground state at a given $\phi / \phi_{0}$ we must sum the contributions from the lowest occupied levels at the considered flux value. For simplicity we ignore spin and assume that a level may be occupied only by one electron. As is well known the current is different for even and for odd numbers of electrons[5]. We first concentrate on the contribution to the total current from the zeroth order levels corresponding to the non-disordered system. For odd $N_{e}$ and for values of flux in

the interval $-\frac{1}{2} \leq \frac{\phi}{\phi_{0}}<\frac{1}{2}$ this contribution, $I^{(0)}$, is obtained by summing the oneelectron currents $(\sqrt{26})$ from $k=-\frac{N_{e}-1}{2}$ to $k=\frac{N_{e}-1}{2}$. The fact that these values of $k$ 
correspond to the $N_{e}$ lowest levels of the spectrum in the considered range of fluxes is easily verified e.g. in the free particle-like limit of (25) where the eigenernergies reduce to intersecting parabolas whose vertices on the $\phi$-axis correspond to integer values of $\phi / \phi_{0}$. One finds in this case[5]

$$
I^{(0)}=\sum_{k=-\left(N_{e}-1\right) / 2}^{\left(N_{e}-1\right) / 2} I_{k}^{0}=-I_{0} \frac{\sin \frac{2 \pi \phi}{N \phi_{0}}}{\sin \frac{\pi}{N}} \simeq-2 \frac{\phi}{\phi_{0}} I_{0}
$$

where

$$
I_{0}=\frac{e v_{F}^{b}}{N}, \text { with } v_{F}^{b}=\frac{2}{\hbar} \sin \frac{\pi N_{e}}{N}
$$

The meaning of the velocity $v_{F}^{b}$ is clear: its value coincides with the quantity $\left.\frac{N}{h} \frac{\partial E_{k}^{0}}{\partial k}\right|_{k=N_{e} / 2}$ which is the band velocity at an energy just above the highest occupied level, that is a velocity close to the velocity at the fermi level. It may also be simply related to a fermi velocity as follows. Consider a non-disordered linear tight-binding chain whose electronic states are described by the equation $-V\left(\varphi_{n+1}+\varphi_{n-1}\right)=E \varphi_{n}$ for a system of $N$ sites. In the continuum limit

$(a \rightarrow 0)$ this equation becomes (with $x=n a)-a^{2} V d^{2} \varphi(x) / d x^{2}=(E-2 V) \varphi(x)$, which is the Schršdinger equation for a free particle of mass $m=\hbar^{2} /\left(2 V a^{2}\right)$. In this limit the fermi velocity for a system of $N_{e}$ electrons is $v_{F}=\hbar k_{F} / n=\frac{2 V}{\hbar} \frac{N_{e} \pi}{N}$ (with $k_{F}=N_{e} \pi /(N a)$ ) the fermi wavenumber). This shows that $v_{F}$ is the limiting value of the band velocity in (40) for low band filling, $N_{e} \pi \ll N$. This discussion shows that $I_{0}$ is the current associated with a single electron of velocity $v_{F}^{b}$ at the fermi level (or of fermi velocity $v_{F}$ for low band filling). This is the well-known manifestation of the strong compensation of the contributions to the persistent current from successive occupied levels of the system, with the result that the total current is approximately equal to the current from the highest occupied level.

Similarly, from the structure of the level spectrum (25), it follows that for even $N_{e}$ states for $1 \leq \phi / \phi_{0}<0$ the $N_{e}$ lowest occupied states correspond to $k=0, \pm 1, \pm 2, \ldots, \pm\left(\frac{N_{e}}{2}-1\right),-\frac{N_{e}}{2}$. In this case one thus obtains[5]

$$
I^{(0)}=-I_{0} \frac{\sin \left[\frac{\pi}{N}\left(\frac{2 \phi}{\phi_{0}}-1\right)\right]}{\sin \frac{\pi}{N}} \simeq-\left(\frac{2 \phi}{\phi_{0}}-1\right) I_{0}
$$

Obviously the strong compensation of contributions from adjacent levels to the pure system current shown by (4041) does not occur when summing the effect of the disorder in (38) for the successive occupied levels. Thus, unlike for the contribution from the non-disordered energy levels, we expect to obtain a reasonable estimate of the effect of the disorder on the averaged current by approximating the discrete sum over occupied levels by an integral. More precisely, we shall use the Euler-MacLaurin summation formula[36]: 


$$
\sum_{k=1}^{n-1} f_{k} \simeq \int_{0}^{n} f(k) d k-\frac{1}{2}[f(0)+f(n)]
$$

where we have included only the leading integrated terms[36].

For odd $N_{e}$ and $-\frac{1}{2} \leq \frac{\phi}{\phi_{0}}<\frac{1}{2}$ where the summation over occupied levels runs over $k=0, \pm 1, \ldots, \pm\left(\frac{N_{e}-1}{2}\right)$ we obtain, by applying (42),

$$
\sum_{k} \frac{1}{\sin \frac{2 \pi}{N}\left(k+\frac{\phi}{\phi_{0}}\right)}=-\frac{1}{2}\left(\frac{1}{\sin r_{+}}-\frac{1}{\sin r_{-}}\right)-\frac{N}{4 \pi} \ln \frac{\left(1+\cos r_{+}\right)\left(1-\cos r_{-}\right)}{\left(1-\cos r_{+}\right)\left(1+\cos r_{-}\right)}
$$

where

$$
r_{ \pm}=\frac{\pi}{N}\left(N_{e}+1 \pm 2 \frac{\phi}{\phi_{0}}\right)
$$

In the case of even $N_{e}$, for $0 \leq \phi / \phi_{0}<1$, the summation over $k$ is over the values $k=0, \pm 1, \ldots, \pm\left(\frac{N_{e}}{2}-1\right)$ and $-\frac{N_{e}}{2}$ and we find

$$
\sum_{k} \frac{1}{\sin \frac{2 \pi}{N}\left(k+\frac{\phi}{\phi_{0}}\right)}=-\frac{1}{2}\left(\frac{1}{\sin s_{+}}+\frac{1}{\sin s_{-}}\right)-\frac{N}{4 \pi} \ln \frac{\left(1+\cos s_{+}\right)\left(1-\cos s_{-}\right)}{\left(1-\cos s_{+}\right)\left(1+\cos s_{-}\right)}
$$

where

$$
s_{ \pm}=\frac{\pi}{N}\left(N_{e} \pm 2 \frac{\phi}{\phi_{0}}\right)
$$

After some simplification of the disorder contributions the expressions for the total averaged current, $\langle I\rangle=\sum_{k}\left\langle I_{k}\right\rangle$ given by (38), (39) and (43444) for odd $N_{e}$ and by (38), (41) and (45-46) for even $N_{e}$, respectively, reduce finally to (with $k_{F}=\frac{\pi N_{e}}{N a}$ )

$$
\begin{aligned}
\langle I\rangle=-I_{0}\left\{\frac{\sin \frac{2 \pi \phi}{N \phi_{0}}}{\sin \frac{\pi}{N}}+\frac{N \varepsilon_{0}^{2}}{8 \sin ^{2} \frac{2 \pi \phi}{\phi_{0}} \sin k_{F} a} .\right. & \\
& {\left.\left[\frac{\cos \left(k_{F} a+\frac{\pi}{N}\right) \sin \frac{2 \pi \phi}{N \phi_{0}}}{\sin ^{2} \frac{2 \pi \phi}{N \phi_{0}}-\sin ^{2}\left(k_{F} a+\frac{\pi}{N}\right)}+\frac{N}{2 \pi} \ln \left(\frac{\sin \left(k_{F} a+\frac{\pi}{N}\right)-\sin \frac{2 \pi \phi}{N \phi_{0}}}{\sin \left(k_{F} a+\frac{\pi}{N}\right)+\sin \frac{2 \pi \phi}{N \phi_{0}}}\right)\right]\right\} } \\
& \text { for odd } N_{e},-\frac{1}{2}<\frac{\phi}{\phi_{0}}<\frac{1}{2}, \phi \neq 0,
\end{aligned}
$$

and 


$$
\begin{gathered}
\langle I\rangle=-I_{0}\left\{\frac{\sin \left[\frac{\pi}{N}\left(\frac{2 \phi}{\phi_{0}}-1\right)\right]}{\sin \frac{\pi}{N}}-\frac{N \varepsilon_{0}^{2}}{8 \sin ^{2} \frac{2 \pi \phi}{\phi_{0}} \sin \frac{\pi N_{e}}{N}} .\right. \\
\left.\left[\frac{\sin k_{F} a \cos \frac{2 \pi \phi}{N \phi_{0}}}{\sin ^{2} \frac{2 \pi \phi}{N \phi_{0}}-\sin ^{2} k_{F} a}-\frac{N}{2 \pi} \ln \left(\frac{\sin k_{F} a-\sin \frac{2 \pi \phi}{N \phi_{0}}}{\sin k_{F} a+\sin \frac{2 \pi \phi}{N \phi_{0}}}\right)\right]\right\} \\
\text { for even } N_{e}, 0<\frac{\phi}{\phi_{0}}<1, \phi \neq \frac{1}{2} .
\end{gathered}
$$

The formulae (47-48) together with (32) and (37) are the main results of the present paper. 


\section{DISCUSSION AND CONCLUDING REMARKS}

In discussing the averaged persistent currents (47) and (48) we consider successively two limits for the so-called band filling factor $N_{e} / N$ : the case of low filling, $\frac{\pi N_{e}}{N} \ll 1, N_{e} \gg 1$, and the half-filled band case, $N_{e}=N / 2$, for even $N$. For $\pi N_{e} \ll N$, one easily verifies that the disorder corrections in (4748) are of opposite signs to those of the perfect systems currents i.e. the disorder suppresses the persistent current. On the other hand, in the half-filled band case, $N_{e}=\frac{N}{2}$, we obtain successively from (47) and (48)

$$
\begin{aligned}
& \langle I\rangle=-I_{0} \frac{2 \phi}{\phi_{0}}\left(1-\frac{N \varepsilon_{0}^{2}}{8 \sin ^{2} \frac{2 \pi \phi}{\phi_{0}}}\right), \\
& \qquad \text { for odd } N_{e},-\frac{1}{2}<\frac{\phi}{\phi_{0}}<\frac{1}{2}, \phi \neq 0
\end{aligned}
$$

and

$$
\begin{aligned}
\langle I\rangle=-I_{0}\left(\frac{2 \phi}{\phi_{0}}-1\right)\left(1-\frac{N \varepsilon_{0}^{2}}{8 \sin ^{2} \frac{2 \pi \phi}{\phi_{0}}}\right) & \\
& \text { for even } N_{e}, 0<\frac{\phi}{\phi_{0}}<1, \frac{\phi}{\phi_{0}} \neq \frac{1}{2},
\end{aligned}
$$

to leading order in $\frac{2 \pi}{N} \frac{\phi}{\phi_{0}}$. The equation (495) show that for the half-filled band cases the effect of the disorder is simply to multiply the perfect system current by a periodic flux dependent reduction factor of period $\phi_{0} / 2$. This reduction factor does not alter the periodicity with period $\phi_{0}$ of the original perfect system current discussed in[5-7]. We recall that the reduction of the current due to the disorder has a simple interpretation in terms of level repulsion caused by the random impurity potential. Consider, for simplicity, the limit where the conducting electrons are free and the energy level spectrum as a function of $\phi / \phi_{0}$ is composed of intersecting parabolas. As is well-known, the one-body impurity potential causes level repulsion which leads to the replacement of the free parabola crossings by avoided crossings. The latter are more flat than the original true crossings, which leads to a reduction of the currents $\left\langle I_{k}\right\rangle=-c \partial\left\langle E_{k}\right\rangle / \partial \phi$ near them, caused by the interaction with the impurities.

The expression (49-50) for weak randomness differ remarkably from the corresponding results of by Cheung et al.[5]. While our results are obtained from an exact solution of the secular equation (17) to second order in the random site energies for weak randomness, those of Cheung et al. [5] follow from an approximate study of their exact eigenvalue equation

$$
R e\left(\frac{1}{t}\right)=\cos 2 \pi \frac{\phi}{\phi_{0}},
$$

obtained from (9) and (10.c) after transforming to a Bloch wave representation as discussed in Sect. I. We recall that $t$ is the complex transmission coefficient at energy $E$ across a disordered linear chain of length $N a$ obtained by cutting the ring open. 
From simple but crude assumptions about the energy dependence of $t$ Cheung et al. derive an expression for the current from a level $E_{k}$ which may be approximated by $I_{k} \simeq I_{0}|t|[5]$. For a half-filled band, assuming that the total current in the presence of disorder is approximately equal to $-\left(2 \phi / \phi_{0}-1\right)$ times the current carried by the last occupied level they obtain[37]

$$
\langle I\rangle=-\left(\frac{2 \phi}{\phi_{0}}-1\right) I_{0}\langle|t|\rangle
$$

where $\langle|t|\rangle$ is the averaged transmission coefficient measured at the fermi level which may be related to the localization leength by using the Landauer formula. For weak disorder $\langle|t|\rangle$ is thus found to decay as $N \varepsilon_{0}^{2}$ (in our notations[38]), with a constant coefficient, from the value one for the ordered system (See Sect. III.C of [5]). In contrast the exact weak disorder effect in (50), which is also proportional to $N \varepsilon_{0}^{2}$, varies strongly with the flux, as $1 / \sin ^{2} \frac{2 \pi \phi}{\phi_{0}}$. The strong flux dependence of the disorder effect in the persistent current (49-50) shows that the approximations used by Cheung et al. [5] in solving (51) are insufficient, at least for the case of weak disorder.

In discussing the magnitude of the effect of the disorder in (49-50) relative to the perfect system current, it is useful to recall the domain of validity of these perturbative expressions. This domain is determined by:

1. the condition, $\left|\varepsilon_{0}\right| \ll 1$, of smallness of the amplitude fluctuations of the site energies relative to the hopping parameter in (2-4)

2. the inequality $\sqrt{\left\langle E^{(1) 2}\right\rangle} \ll \Delta$, which expresses that the typical shift of energy levels due to disorder must be small compared to the spacing $\Delta$ of energy levels at zero flux at the fermi surface[5,7]. From (25) we have $\Delta=\frac{4 \pi}{N} \cdot \sin k_{F} a$, and by using (31) and (34) this condition reduces to

$$
\sqrt{N}\left|\varepsilon_{0}\right| \ll 4 \pi
$$

in the half-filled band case. This implies that within the perturbative domain the disorder term in the brackett of (50) may be enhanced to values of order unity by the factor $1 / \sin ^{2} \frac{2 \pi \phi}{\phi_{0}}$, for fluxes sufficiently close to multiples of $\phi_{0} / 2$. For odd $N_{e}$ this leads to a strongly enhanced suppression of the current near $\phi= \pm \frac{\phi_{0}}{2}$ while for even $N_{e}$ a similar enhanced suppression occurs near $\phi=0$ and near $\phi= \pm \phi_{0}$. Such large effects of a weak disorder may exist only for mesoscopic rings since e.g. for $\left|\varepsilon_{0}\right|=0.1$ values of $N$ compatible with (53) would be restricted to $N \sim 10^{2}$.

We conclude by suggesting that important modifications of the amplitude of persistent currents in discrete rings due to the interplay of disorder and magnetic flux may also exist for more realistic multichannel disordered rings as well as for models incorporating Coulomb interaction. In view of the persisting disagreements between calculated and measured persistent currents in metallic rings (see Sect. ÊI) such effects would seem to deserve further detailed study. 


\section{References}

[1] M. BŸttiker, Y. Imry, and R. ÊLandauer, Phys.Lett. 96A, 365 (1983).

[2] L.P. Levy, G. Dolan, J. Dunsmuir, and H. Bouchiat, Phys.Rev.Lett. 64, 2074 (1990).

[3] V. Chandrasekkar, R.A. Welb, M.J. Brady, M.B. Ketchen, W.J. Galager, and A. Kleinsasser, Phys.Rev.Lett. 67, 3578 (1991).

[4] D. Mailly, C. Chapelier, and A. Beno"t, Phys.Rev.Lett. 70, 2020 (1993).

[5] H.F. Cheung, Y. Gefen, E.K. Riedel, and W.H. Shih, Phys.Rev. B37, 6050 (1988).

[6] N. Trivedi, and D.A. Browne, Phys.Rev. B38, 9581 (1988).

[7] H.F. Cheung, Y. Gefen, and E.K. Riedel, IBM J.Res.Develop. 32, 359 (1988).

[8] H.F. Cheung, and E.K. Riedel, Phys.Rev. B40, 9498 (1989).

[9] H.F. Cheung, E. Riedel, and Y. Gefen, Phys.Rev.Lett. 62, 587 (1989).

[10] O. Entin-Wohlmann, and Y. Gefen, Europhys.Lett. 5, 447 (1989).

[11] H. Bouchiat, and G. Montambaux, J.Phys. (Paris) 50, 2695 (1989).

[12] G. Montambaux, H. Bouchiat, D. Sigeti, and R. Friesner, Phys.Rev. B42, 7647 (1990).

[13] A. Schmid, Phys.Rev.Lett. 66, 80 (1991).

[14] B.L. Altshuler, Y. Gefen, and Y. Imry, Phys.Rev.Lett. 66, 88 (1991).

[15] V. Ambegaokar, and U. Eckern, Phys.Rev.Lett. 65, 381 (1990).

[16] F. von Oppen, and E.K. Riedel, Phys.Rev.Lett. 66, 84 (1991).

[17] H. Bouchiat, G. Montambaux, and D. Sigeti, Phys.Rev. B44, 1682 (1991).

[18] O.N. Dorokhov, Zh.Eksp.Teor.Fiz. 101, 966 (1992) [Sov.Phys.: JETP 74, 518 (1992)].

[19] A. Altland, S. Iida, A. MŸller-Groeling, and H.A. Weidenm Ÿller, Europhys.Lett. 20, 155 (1992).

[20] A. MŸller-Groeling, H.A. WeidenmŸller, and C.H. Lewenkopf, Europhys.Lett. 22, 193 (1993).

[21] M. Abraham, and R. Berkovits, Phys.Rev.Lett. 70, 1509 (1993).

[22] P. Kopietz, Phys.Rev.Lett. 70, 3123 (1993).

[23] A. MŸller-Groeling, and H.A. WeidenmŸller, Phys.Rev. B49, 4752 (1994).

[24] G. Bouzerar, D. Poilblanc, and G. Montambaux, Phys.Rev. B49, 8258 (1994). 
[25] H. Kato, and D. Yoshioka, Phys.Rev.B R50, 4943 (1994).

[26] G. Vignale, Phys.Rev. B50, 7668 (1994).

[27] W.C. Tan, and J.C. Inkson, Phys.Rev. B60, 5626 (1999).

[28] X. Chen, Z.Y. Deng, W. Lu, and S.C. Shen, Phys.Rev. B61, 2008 (2000).

[29] U. Eckern, and P. Schwab, Adv.in Phys. 44, 387 (1995).

[30] Y. Imry, Introduction to Mesoscopic Physics (Oxford University Press, Oxford, 1997).

[31] K. Efetov, Supersymmetry in Disorder and Chaos (Cambridge University Press, Cambridge, 1997).

[32] N. Byers, and C.N. Yang, Phys.Rev.Lett. 7, 46 (1961).

[33] F. Bloch, Phys.Rev. 137, , A787 (1965); ibid. 166, 415 (1968); ibid. B2 , 109 (1970).

[34] J.M. Luck, Systemes Desordonnes Unidimensionnels (Alaa, Saclay, 1992).

[35] J.B. Pendry, Adv.Phys. 43, 461 (1994).

[36] J. Mathews, and R.L. Walker, Mathematical Methods in Physics (W.A. Benjamin, Menlo Park, California, 1973).

[37] The discussion of the persistent current for weak disorder for $\phi / \phi_{0}=1 / 4$ by Cheung et al.[5] may be readily extended to arbitrary typical values of flux. In this case one assumes that, like for the pure system, the total current is given by $-\left(2 \phi / \phi_{0}-1\right)$ times the current of the last occupied level. This generalizes the corresponding assumption of Cheung et al. for the case $\phi / \phi_{0}=1 / 4$.

[38] The relation between the rectangular distribution of width $W$ for the site energies used in [5] and the gaussian parameter $\varepsilon_{0}$ is obtained by identifying the second moment of this distribution with $\varepsilon_{0}^{2}=\left\langle\varepsilon_{j}^{2}\right\rangle, j=1, \ldots, N$. This yields $\varepsilon_{0}^{2}=W^{2} / 12$. 